\title{
To Determine Cutoff Value of Triglycerides to HDL Ratio in Cardio Vascular Risk Factors
}

\author{
Qurat UI Ain ${ }^{1 *}$, Naveed Asif ${ }^{1}$, Mehwish Gilani ${ }^{1}$, Noreen $^{1}$, Waqas Sheikh ${ }^{1}$ and Aammad Akram ${ }^{2}$ \\ ${ }^{1}$ Department of Chemical Pathology and Endocrinology, Armed Forces Institute of Pathology, Rawalpindi, Pakistan \\ ${ }^{2}$ Military Hospital Rawalpindi, Rawalpindi, Pakistan
}

"Corresponding author: Qurat UI Ain, Department of Chemical Pathology and Endocrinology, Armed Forces Institute of Pathology, Rawalpindi, Pakistan, Tel: +923333643355; E-mail: draaneiseera@gmail.com

Received Date: May 15, 2018; Accepted Date: May 26, 2018; Published Date: Jun 04, 2018

Copyright: () 2018 Ain QU, et al. This is an open-access article distributed under the terms of the Creative Commons Attribution License, which permits unrestricted use, distribution, and reproduction in any medium, provided the original author and source are credited.

\author{
Abstract \\ Objective: This study was conducted with the aim of determining the cutoff value for Triglycerides to HDL ratio in \\ adults with cardiovascular risk factors.
}

Study design: The study design was cross sectional which is observational cross sectional.

Place and duration of study: Department of Chemical Pathology and Endocrinology, Armed Forces Institute of Pathology Rawalpindi from January 2018-June 2018.

Methodology: This study was a cross-sectional study conducted after the Institutional Review Board's (IRB) approval at Armed Forces Institute of Pathology from January 2018-June 2018. Data was collected from 354 patients. Inclusion criteria included adults with 19-50 years of age. Patient with comorbidity like cancer, tuberculosis, bed ridden patients were excluded from study. Sampling technique was simple random sampling which was done by simply picking participants through random numbers.

Results: Out of a total 354 patients selected, $269(71.5 \%)$ were females while $86(22.9 \%)$ were males with mean age of $37 \pm 11.64$ years with range of $22-60$ years of age group. A cut off of 1.0 for Triglycerides to HDL-C ratio was able to identify participants with cardio metabolic risk factors (obesity, hypertension, diabetes). The AUC of ROC for the ability of TG/HDL-C ratio to predict cardio metabolic risk factors was significant with coordinates of $0.68 \pm 1.60$ ( $p$-value=0.03). Particularly with a cutoff point of 1.0 it showed a sensitivity of $76 \%$ while specificity was $64 \%$ for early diagnosis of cardiovascular risks factors.

Conclusion: This study concluded that using 1.0 as an optimal cutoff of TG/HDL ratio can be used as a predictor and an early marker for cardio metabolic risk factors.

Keywords: High density lipoprotein; Cardiovascular disease; TG/HDL Ratio; Obesity

\section{Introduction}

Cardio vascular diseases (CVD) are the leading cause of death and are the most common cause of mortality due to non-communicable diseases like myocardial infarction, endocarditis and others [1]. Mortality due to CVD is common in developed countries as well. It is the main cause of triple burden of disease in developing and middle income countries [2]. Obesity, hypertension, dyslipidemia are important health problems that have reached epidemic proportions worldwide. Several studies of evidence have highlighted an alarming association between childhood, adolescence, adult obesity and development of CVD [2,3]. Increase body weight as compared to the mean body weight of that age group during childhood, as well as in adults lead to metabolic and inflammatory alterations, which in turn may cause changes in the arterial wall and contribute in development of cardiovascular events during adulthood [4]. Many metabolic and inflammatory factors seem to be implicated in the pathogenesis of atherosclerosis. In particular, insulin resistance (IR) represents an important link between obesity and the associated cardiovascular risk and it has been suggested as one of the first mechanisms involved in the development of endothelial dysfunction in obese adults [5]. In addition, oxidative stress and inflammatory reaction at molecular level, related to an increased adipose tissue, are additional players in the development of the atherosclerotic plaque. The main aim of this study was to the association between TG: HDL-C ratio and cardiovascular risk factors as well as early signs of vascular damage in adults [6]. Lipid metabolic disorders are proven pathogenesis of atherosclerosis and any defect in lipid disorders can increase risk for CVD [7]. Hyperlipidemia is increasing in European, Western countries as well as in Asian population. High level of Low density Lipoprotein cholesterol (LDL-C) is well established in CVD, while role of High density lipoprotein (HDL-C) and triglycerides is controversial [8]. Some studies demonstrate that hypertriglyceridemia is an independent factor for CVD and has been proven as a stronger risk factor for the heart disease [9]. Initially Triglycerides and HDL ratio was proposed by Gazeino et al. and it was suggested that it is a highly predictive independent factor for CVD. Cardiovascular disorders are associated with heart and vascular system. In heart it is mainly concerned with coronary heart disease, rheumatic heart disease and other conditions. Four out of five 
cerebrovascular diseases are due to heart attack and strokes [10,11] Plasma ratio of triglyceride (TG)/HDL cholesterol (HDL-C) provide a simple way to identify apparently healthy insulin resistant persons with increased cardio metabolic risk factors. However, there is evidence that the actual values of the ratio that best identifies such individuals will vary as a function of racial/ethnic background. More recently, it has also been shown that the most useful TG/HDL-C cut-point to identify cardio metabolic risk is not the same in men and women. It is also considered as an easy and rapid indicator especially in the context of primary health care. Recent analysis have shown that this ratio is a potent predictor of the development of coronary heart disease and is directly correlated with plasma B-type LDL cholesterol levels. Therefore, current study was aimed to find cutoff value of TG/HDL ratio in Pakistani population as a predictor for early CVD diseases $[12,13]$.

\section{Material and Methods}

This study was a cross-sectional study conducted after the Institutional Review Board's (IRB) approval at Armed Forces Institute of Pathology from January 2018-June 2018. Data was collected from 354 patients from different socioeconomic status who came to Armed Forces Institute of Pathology in CMH Rawalpindi after informed consent. The Research Ethics Committee of the AFIP approved the study protocol. Inclusion criteria included adults with age of 19-60 years. Patients with cancer, tuberculosis, bed ridden patients were excluded from study. Sampling technique was simple random sampling which was done by simply picking participants through random numbers. A structured, standardized and pre-tested questionnaire was applied in the pilot study. The independent variables evaluated at the first meeting were socioeconomic and demographic factors (age, gender, marital status, and years of schooling and economic class), lifestyle (smoking, alcohol consumption and sedentary behavior) and presence of morbidities (heart disease, dyslipidemia, hypertension, diabetes, presence of depressive symptoms). Next, the anthropometric data for obesity classification (weight, height and waist circumference) was measured. Anthropometric measured through In Body Composition Analysis System in which all parameters sort out for all patients (Body mass index, Visceral Fats, Weight, Height, Percent Body fats, skeletal muscle mass and ideal body weight) which works on principle of bioelectrical impedance analysis (BIA) measured body water by obtaining impedance index. BMI had been divided into three main categories defined by World Health Organization (Normal, Over weight and obese). Participants who were having BMI of less than 25 were considered normal, while BMI of 25.5-30 were considered overweight and more than 30 were considered as obese. On the basis of visceral fats, subjects were again divided into two groups; normal visceral fats were (1-9\%) while high visceral fats were (10-20\%). At the second meeting a biochemical evaluation was performed. Participants were called after an overnight fast of 12 hours for the evaluation of diabetic profile (blood glucose fasting), lipid profile (cholesterol, HDLC, LDL-C, Triglycerides, VLDL-C) and serum insulin. To ensure the accuracy and precision of the test results, all preanalytical, analytical, and post analytical precautions were taken into consideration. Instruments, personnel, and procedure validation were carried out through an internal quality control (QC) program with the calculation of standard deviations (SD) and coefficients of variation (CV). Quality control material was traceable to National institute of standardization (NIST). Three milliliters of venous blood was collected from antecubital vein in yellow topped serum separator tube for lipid profile and serum insulin each. For fasting glucose $3 \mathrm{ml}$ blood was taken in grey topped sodium fluoride vacutainers. Samples were sent to laboratory and centrifuged within 2 hours. Sample for lipid profile and fasting glucose were analyzed on fully automated random access chemistry analyzer Advia 1800 by spectrophotometry. While serum insulin fasting was analyzed by immunoassay on Advia Xp by competitive sandwich immunoassay. HOMA-IR calculated by fasting plasma glucose value and serum insulin fasting value divided by 22.5 and considered insulin resistance if this value $>2.5$ while Whole body insulin sensitivity index calculated by using $10,000 /$ fasting plasma glucose $\times$ serum fasting insulin $\times$ mean plasma glucose $\times$ mean serum insulin. QUICKI was calculated by using the formula $1 /$ \{log (fasting insulin $\mathrm{uU} / \mathrm{ml})+\log$ (fasting glucose $\mathrm{mg} / \mathrm{dl})\}$. Data analyzed using SPSS version 24. For descriptive statistics Mean \pm SD computed for quantitative variables while frequency was calculated for qualitative variables. ROC curve computed to determine cutoff level among cardiac risk factors.

\section{Result}

A total of 354 sample size was analyzed after getting approval from ethical review board of Armed Forces Institute of Pathology Rawalpindi, out of which 269 (71.5\%) were females while $86(22.9 \%)$ were males with mean age of $37 \pm 11.64$ years with range of $22-60$ year of age group. Two hundred and eighty three (75.3\%) participants belonged to urban area while $72(19.1 \%)$ belonged to rural areas. There were $152(40 \%)$ patients with primary hypertension while 203 (54\%) had no complaints of hypertension. Most of the patients with hypertension had duration of 2-3 years of disease and were on antihypertensive medicines. There were 155 (41.2\%) participants who were diabetics and previously diagnosed on the basis of fasting plasma glucose, OGTT with $75 \mathrm{mg}$ anhydrous glucose and HbAlc while 200 (53.2\%) were non-diabetic. Out of 354, 16 (18.9\%) were with BMI less than 25, $94(25 \%)$ with BMI 25.5-30 and comes in the range of overweight, while 192 (51.1\%) were with BMI more than 30 and come in range of obese. Participants with visceral fats 1-9\% were $48(12.8 \%)$ and with visceral fats $9-20 \%$ were 307 (81.6\%). Patient with coronary heart diseases diagnosed with Angiography were 64 (17\%) while those who didn't have heart disease were 291 (77.4\%). Mean \pm SD of all quantitative variables (Age, Height, Weight, Body Mass index, Percent body fats ,Visceral fats, LDL-C, HDL-C, Triglycerides, HOMA-IR, QUICKI and TG/HDL-C ratio and WBISI) with respect to male and female were computed (Table 1).

\begin{tabular}{|c|c|c|}
\hline \multicolumn{2}{|l|}{ Variables } & \multicolumn{2}{|l|}{$\begin{array}{l}\text { Male } \\
\text { (Mean } \pm \text { SD) }\end{array}$} & $\begin{array}{l}\text { Female } \\
\text { (Mean } \pm \text { SD) }\end{array}$ \\
\hline Age & $38 \pm 11.0$ & $37 \pm 13.2$ \\
\hline Height $(\mathrm{cm})$ & $170.1 \pm 3.64$ & $160.3 \pm 6.22$ \\
\hline Weight $(\mathrm{Kg})$ & $85.3 \pm 15.6$ & $82.3 \pm 11.5$ \\
\hline BMI & $29.4 \pm 4.9$ & $31.5 \pm 15.6$ \\
\hline Visceral Fats & $14.0 \pm 3.8$ & $18.9 \pm 1.96$ \\
\hline Skeletal muscle mass & $29.9 \pm 4.3$ & $23.8 \pm 2.4$ \\
\hline Triglycerides (mmol/l) & $1.47 \pm 0.52$ & $1.44 \pm 1.2$ \\
\hline LDL-C (mmol/l) & $2.25 \pm 1.48$ & $2.38 \pm 0.50$ \\
\hline HDL-C (mmol/) & $1.2 \pm 0.66$ & $1.0 \pm 0.23$ \\
\hline Insulin Fasting (IU/ml) & $12.9 \pm 6.5$ & $21.0 \pm 9.1$ \\
\hline
\end{tabular}




\begin{tabular}{|c|c|c|}
\hline HOMA-IR & $0.92 \pm 1.61$ & $2.0 \pm 2.42$ \\
\hline QUICKI & $0.70 \pm 0.45$ & $0.53 \pm 0.61$ \\
\hline TG/HDL Ratio & $1.41 \pm 0.71$ & $1.42 \pm 1.45$ \\
\hline
\end{tabular}

Table 1: Descriptive statistics of quantitative variables (In male and female).

ROC curve was used to calculate Triglycerides to HDL-C ratio for cardiovascular risk factors. At a cutoff of 1.0 for Triglycerides to HDL$\mathrm{C}$ ratio was able to identify participants with cardio metabolic risk factors. The AUC of ROC for the ability of TG/HDL-C ratio to predict the cardio metabolic risk factors was significant with coordinates of $0.68 \pm 1.60$ ( $\mathrm{p}$ value $=0.03$ ). Particularly with the cutoff point of 1.0 it showed a sensitivity of $76 \%$ while a specificity of $64 \%$ for the early diagnosis of cardiovascular risks (obesity, hypertension ,diabetes) (Figure 1 and Table 2).

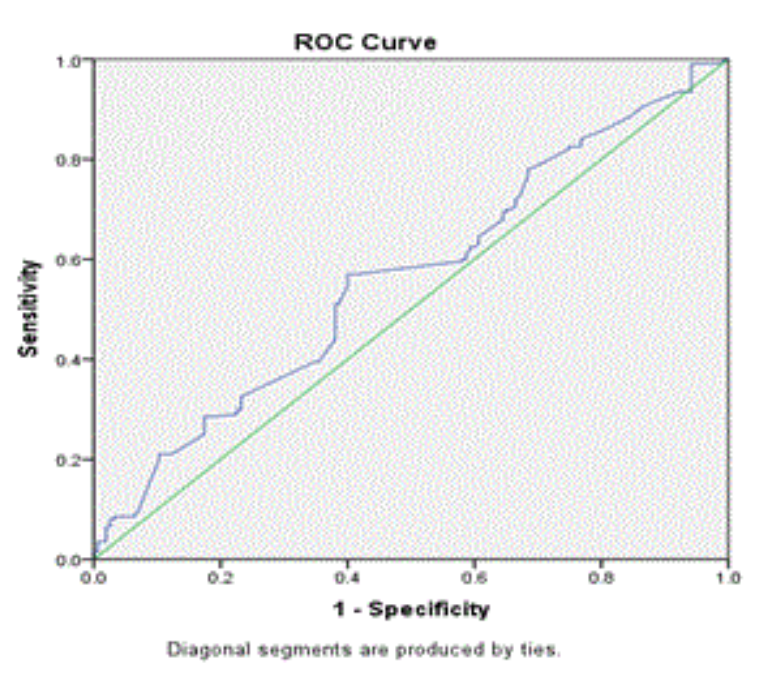

Figure 1: ROC curve computed in SPSS software 24 for optimal cutoff level of TG/HDL ratio with cardiac risk factors (obesity, HTN, Diabetes) with table of cut offs along with specificity and sensitivity. The cutoff determine by the area near the highest sensitivity and specificity area.

\begin{tabular}{|c|c|c|c|c|c|c|}
\hline Cut off & AUC $^{*}$ & Sensitivity & Specificity & PPV & NPV & Accuracy \\
\hline 1.0 & 0.567 & $75 \%$ & $63 \%$ & $72 \%$ & $67 \%$ & $71 \%$ \\
\hline
\end{tabular}

Table 2: Sensitivity and specificity at cutoff level of $1.0 \mathrm{mmol} / \mathrm{L}$ (Area under curve)*.

\section{Discussion}

This study was aimed to set cutoff value of TG/HDL-C ratio as a predictor and potent marker of early diagnosis of cardio metabolic risk factors. TG/HDL-C ratio was associated with cardiovascular risk factors and this finding is relevant to current study which shows strong association of TG/HDL C ratio with cardiac risk like hypertension and diabetes [14]. Study conducted by Kayani et al. in Armed Forces Institute of cardiology Pakistan proved that age group affected by Cardiovascular diseases is $50-59$ year in males while in females it is more common in age group of 60-69 year of age which is conflicted in current study that the most common age group affected in male is 45-50 year while regarding female age group affected is 60 years, it correlates with the findings of study conducted by Kayani et al. [14] Several studies in past proved strong association of TG/HDL-C Ratio in obese children for early diagnosis of atherosclerosis. The main focus of TG/HDL-C ratio is atherosclerosis by decrease level of HDL-c and higher value of Triglycerides value [15]. However in adults with increased risk of cardiac and metabolic factors it is not always reflect in normal lipid profile that's why TG/HDL C ratio considered more effective in early prediction of cardio metabolic risk factors. Results of this study are consistent with the study conducted in Italy by Mohan et al. in 2014 showed that cutoff limit for TG/HDL C ratio 1.12 while in our study the cut off limit is 1.0. In the same study the sensitivity and specificity at cutoff limit of $1.1 \mathrm{mmol} / \mathrm{L}$ is $81 \%$ and $49 \%$ respectively, while in current study sensitivity was $76 \%$ and specificity was $64 \%$ which is also relevant to results of study conducted in Italy in 2014. In a study by Di Beito et al. a finding that TG/HDL C ratio is strong predictor and early useful index for cardiac remolding which correlate with current study findings that extend association of different cardio metabolic risk factors with TG/HDLC ratio $[16,17]$. Kayani et al. also proved that risk factors which can cause cardiac diseases in future are hypertension, obesity and diabetes in the present study extend association among these risk factors with TG/HDLC ratio. In a study conducted in united Kingdom by Samiul A. Mostafa extend association of Insulin resistance with the TG/HDL ratio which correlate with the results of present study which proved strong association between HOMA-IR marker for Insulin resistance with TG/HDL C ratio [17]. This study acknowledged the importance of TG/HDL C ratio importance as a marker to detect cardiovascular risk factors and this study gives an optimal cutoff of TG/HDL-C ratio for early detection of complication of hypertension, diabetes and obesity. This study also has certain limitations like cross sectional study design is not enough for temporal association while small sample size and hospital based study design limit the generality of results that's why need more advance and prospective study design required to prove the fact.

\section{Conclusion}

This study concluded that using 1.0 as an optimal cutoff of TG/HDL ratio can be used as a predictor and an early marker for cardio metabolic risk factors. TG/HDL ratio is comparative better and potent biomarker for early diagnosis of cardio vascular disease with simple lipid profile (LDL, HDL, TG). TG/HDL ratio at this cutoff can detect early cardiac disease although with normal lipid profile.

\section{Declaration of Interest}

The authors declare that there is no conflict of interest that could be perceived as prejudicing the impartiality of the research reported.

\section{Funding Resource}

This research did not receive any specific grant from any funding agency in the public, commercial or not-for-profit sector.

\section{Acknowledgement}

All authors would like to extend thanks to participants of research and staff who were involved directly or indirectly. 


\section{References}

1. Gaziano JM, Hennekens CH, O’Donnell CJ, Breslow JL, Buring JE (1997) Fasting triglycerides, high-density lipoprotein, and risk of myocardial infarction. Circulation 96: 2520-2525.

2. Rathmann W, Giani G (2004) Global Prevalence of Diabetes: Estimates for the Year 2000 and Projections for 2030. Diabetes care 27: 2568-2569.

3. Murguía-Romero M, Jiménez-Flores JR, Sigrist-Flores SC, EspinozaCamacho MA, Jiménez-Morales M, et al. (2013) Plasma triglyceride/ HDL-cholesterol ratio, insulin resistance, and cardiometabolic risk in young adults. Journal of lipid research 54: 2795-2799.

4. Martins MV, Souza JD, Martinho KO, Franco FS, Tinôco AL (2017) Association between triglycerides and HDL-cholesterol ratio and cardiovascular risk factors among elderly persons receiving care under the family health strategy of Viçosa, Minas Gerais. Brazilian Journal of Geriatrics and Gerontology 20: 236-243.

5. de Giorgis T, Marcovecchio ML, Di Giovanni I, Giannini C, Chiavaroli V, et al. (2014) Triglycerides-to-HDL ratio as a new marker of endothelial dysfunction in obese prepubertal children. European journal of endocrinology 170: 173-180.

6. Giannini C, Santoro N, Caprio S, Kim G, Lartaud D, et al. (2011) The triglyceride-to-HDL cholesterol ratio: association with insulin resistance in obese youths of different ethnic backgrounds. Diabetes care 34: 1869-1874.

7. Salazar MR, Carbajal HA, Espeche WG, Leiva Sisnieguez CE, Balbin E, et al. (2012) Relation amongthe plasma triglyceride/high-density lipoprotein cholesterol concentrationratio, insulin resistance, and associated cardio-metabolic riskfactors in men and women. The American Journal of Cardiology 109: 1749-1753.

8. Di Bonito P, Moio N, Scilla C, Cavuto L, Sibilio G, et al. (2012) Usefulness of the high triglyceride-to-HDL cholesterol ratio to identify cardiometabolic risk factors and preclinical signs of organ damage in outpatient children. Diabetes care 35: 158-162.

9. D’Adamo E, Marcovecchio ML, Giannini C, De Giorgis T, Chiavaroli V, et al. (2013) Improved oxidative stress and cardio-metabolic status in obese prepubertal children with liver steatosis treated with lifestyle combined with Vitamin E. Free radical research 47: 146-153.

10. Piepoli MF, Hoes AW, Agewall S, Albus C, Brotons C, et al. (2016) 2016 European Guidelines on cardiovascular disease prevention in clinical practice: The Sixth Joint Task Force of the European Society of Cardiology and Other Societies on Cardiovascular Disease Prevention in Clinical Practice (constituted by representatives of 10 societies and by invited experts) Developed with the special contribution of the European Association for Cardiovascular Prevention \& Rehabilitation (EACPR). Atherosclerosis 252: 207-274.

11. Perk J, De Backer G, Gohlke H, Graham I, Reiner E, et al. (2012) European Guidelines on Cardiovascular Disease Prevention in Clinical Practice. International journal of behavioral medicine 19: 403.

12. Levi F, Chatenoud L, Bertuccio P, Lucchini F, Negri E, et al. (2009) Mortality from cardiovascular and cerebrovascular diseases in Europe and other areas of the world: an update. European Journal of Cardiovascular Prevention \& Rehabilitation 16: 333-350.

13. da Luz PL, Cesena FH, Favarato D, Cerqueira ES (2005) Comparison of serum lipid values in patients with coronary artery disease at $<50,50$ to 59, 60 to 69, and >70 years of age. American Journal of Cardiology 96: 1640-1643.

14. Kayani AM, Bakht N, Munir R, Abid I (2011) The mosaic of CVD risk factors-A study on 10,000 Pakistani cardiac patients. CVD Prevention and Control 6: 1-7.

15. Nguyen DM, El-Serag HB (2010) The epidemiology of obesity. Gastroenterology Clinics 39: 1-7.

16. Palomba S, Russo T, Orio F Jr, Sammartino A, Sbano FM, et al. (2004) Lipid, glucose and homocysteine metabolism in women treated with a GnRH agonist with or without raloxifene. Human Reproduction 19: 415-421.

17. Mustafa SA, Davies MJ, Morris DH, Yates T, Srinivasan BT, et al. (2012) The association of the triglyceride-to-HDL cholesterol ratio with insulin resistance in White European and South Asian men and women. PLoS One 7: e50931. 